\title{
Resting State Brain Network Disturbances Related to Hypomania and Depression in Medication-Free Bipolar Disorder
}

\author{
Jeffrey M Spielberg ${ }^{1,2}$, Erik B Beall ${ }^{3}$, Leslie A Hulvershorn ${ }^{4}$, Murat Altinay ${ }^{5}$, Harish Karne ${ }^{5}$ and Amit Anand ${ }^{*, 5}$ \\ 'Department of Psychological and Brain Sciences, University of Delaware, Newark, DE, USA; ${ }^{2}$ Neuroimaging Research for Veterans Center, VA \\ Boston Healthcare System, Boston, MA, USA; ${ }^{3}$ Imaging Institute, Cleveland Clinic Foundation, Cleveland, OH, USA; ${ }^{4}$ Department of Psychiatry, \\ Indiana University School of Medicine, Indianapolis, IN, USA; ${ }^{5}$ Center for Behavioral Health, Cleveland Clinic Foundation, Cleveland, OH, USA
}

\begin{abstract}
Research on resting functional brain networks in bipolar disorder (BP) has been unable to differentiate between disturbances related to mania or depression, which is necessary to understand the mechanisms leading to each state. Past research has also been unable to elucidate the impact of BP-related network disturbances on the organizational properties of the brain (eg, communication efficiency). Thus, the present work sought to isolate network disturbances related to BP, fractionate these into components associated with manic and depressive symptoms, and characterize the impact of disturbances on network function. Graph theory was used to analyze resting functional magnetic resonance imaging data from 60 medication-free patients meeting the criteria for BP and either a current hypomanic $(n=30)$ or depressed $(n=30)$ episode and 30 closely age/sex-matched healthy controls. Correction for multiple comparisons was carried out. Compared with controls, BP patients evidenced hyperconnectivity in a network involving right amygdala. Fractionation revealed that (hypo)manic symptoms were associated with hyperconnectivity in an overlapping network and disruptions in the brain's 'small-world' network organization. Depressive symptoms predicted hyperconnectivity in a network involving orbitofrontal cortex along with a less resilient global network organization. Findings provide deeper insight into the differential pathophysiological processes associated with hypomania and depression, along with the particular impact these differential processes have on network function.

Neuropsychopharmacology (2016) 41, 3016-3024; doi: I0.1038/npp.2016.1 I2; published online 20 July 2016
\end{abstract}

\section{INTRODUCTION}

Bipolar disorder (BP) is a chronic and debilitating illness defined by episodes of (hypo)mania and depression, and the neural mechanisms triggering these shifts remain unknown (Blumberg, 2012). Although other disorders include episodes of extreme affect (eg, major depressive disorder (MDD)), $\mathrm{BP}$ is uniquely characterized by shifts into two states of seemingly opposing affective valence. Thus, understanding BP-related neural circuitry requires not only identifying disturbances common across mood states but also fractionating disturbances into components characteristic of each state, potentially providing insight into the mechanisms by which each mood state is triggered. In the present study, we examined symptoms continuously, which does not depend on the mood state currently experienced by an individual (eg, manic $v s$ hypomanic). Thus, we refer to symptoms of mania, which occur in both manic and hypomanic episodes, as (hypo)manic symptoms.

*Correspondence: Dr A Anand, Center for Behavioral Health, Cleveland Clinic Foundation, Cleveland Clinic Main Campus, 9500 Euclid Avenue P57, Cleveland, OH 44I95, USA, Tel: + I 2166365860 , Fax: + I 216445 0827, E-mail: ANANDA@ccf.org

Received 30 March 20 6; revised 22 June 20 16; accepted 23 June 20 I6; accepted article preview online 30 June 2016
A large body of BP research implicates dysfunction in brain networks supporting emotion regulation (Strakowski et al, 2012; Brady et al, 2014; Wessa et al, 2014). Metaanalytic evidence indicates that structures involved in topdown control (eg, ventrolateral prefrontal cortex (vlPFC)) are hypoactive, whereas structures crucial for bottom-up affective salience (eg, amygdala) are hyperactive (Chen et al, 2011; Houenou et al, 2011; Delvecchio et al, 2012). Imbalance between systems, potentially reflected in disturbed brain network connectivity, could lead to (hypo)mania and depression.

Mounting evidence supports the existence of BP-related disturbances in functional networks (mapped via restingstate fMRI) (Vargas et al, 2013). Findings have been inconsistent, potentially due to differences in participant's current affective state and because each study examined only a small subset of possible connections. In euthymic patients, increased/decreased amygdala connectivity has been observed with medial (mPFC)/dorsolateral PFC (Anticevic et al, 2013), respectively, along with increased amygdala-vlPFC coupling (Torrisi et al, 2013). Patients in manic episodes exhibited greater $\mathrm{mPFC}$ coupling with insula and vlPFC (Chai et al, 2011), and reduced coupling was observed between ventromedial PFC and regions in the 
default mode network in patients in manic/mixed episodes (Öngür et al, 2010).

Thus, evidence supports the existence of functional network disturbances in $\mathrm{BP}$, but the nature of such disturbances remains poorly understood. Although impressive, several shortcomings in these studies may explain these inconsistencies. For example, the majority did not examine mania and depression simultaneously, making it impossible to parse BP-related disturbances (i) specific to (hypo)mania, (ii) specific to depression, or (iii) present across states. Furthermore, participants were taking psychotropic medications, which can impact fMRI (Anand et al, 2007), making it difficult to disentangle the effects of pathology $v s$ medication.

Finally, the methodology used in these studies has several key limitations. First, each study examined connectivity with only a small set of 'seed' regions (and different sets across studies), potentially overlooking important connections. Furthermore, these methods examined only pairwise coupling, ignoring the role of that connection within the greater network. Most importantly, these methods do not provide insight into whether $\mathrm{BP}$ is associated with restructuring of the functional organization of networks. For example, do individuals with BP have a network organization with worse communication efficiency? Thus, a comprehensive understanding of network disturbances associated with BP generally, and (hypo)mania and depression in particular, remains unknown.

To address these issues, we examined medication-free patients in hypomanic/manic and depressed episodes using graph theory (only two hypomanic/manic patients were in a full manic episode, the rest in hypomanic episodes). Graph theory can elucidate key organizational properties of the global network and the function of regions therein. Graph property categories include: Functional Segregation-how optimized the network is for specialized processing, Functional Integration-how well the network can combine information across distributed regions, and Resiliencevulnerability of the network to disruption. Thus, graph theory can delineate the functional mechanisms by which altered network structure contributes to BP generally and (hypo)mania and depression specifically.

To date, only one BP study has used the graph theory (Leow et al, 2013). Examination of white matter tracts in medicated euthymic patients revealed decreased global Integration and Segregation of the hippocampus and PCC. Thus, these methods have proven useful in the study of structural networks in euthymic BP, suggesting that they will prove useful in characterizing functional networks in (hypo) manic and depressed BP.

We predicted BP participants ( $v s$ healthy controls) would evidence disturbed amygdala-vlPFC connectivity and decreased global Integration. We expected fractionation of BP-related networks to reveal different sets of disturbances for (hypo)manic and depressive symptoms, but did not make specific predictions, given the dearth of research examining these affective states concurrently.

\section{MATERIALS AND METHODS}

\section{Participants}

Participants (aged $=18-60$ years) were recruited via the Indiana University Hospital outpatient psychiatry clinic and community advertisement. Patients were included if they satisfied the DSM-IV-TR criteria for BP and a current (hypo) manic or depressed episode (both BP I and II included, see Supplementary Table 1). Diagnoses were determined via Mini International Neuropsychiatric Interview (Sheehan et al, 1998) and a clinical interview by a psychiatrist (AA). Patients were rated on the Hamilton Depression Rating Scale (HAMD) (Hamilton, 1960) and Young Mania Rating Scale (YMRS) (Young et al, 1978) during both screening and scanning day. Depressed participants were required to have HAMD $\geqslant 15$ and YMRS $\leqslant 10$ and (hypo)manic participants to have $\mathrm{HAMD} \leqslant 12$ and $\mathrm{YMRS} \geqslant 12$ on scanning day to ensure they continued to be in the mood episode in which they were enrolled.

All participants were medication-free (including 'rescue' medications, eg, benzodiazepines) for $\geqslant 2$ weeks before study inclusion and many for much longer (see Supplementary Material for details regarding medication status). Patients were largely moderately depressed or hypomanic. Although participants were currently medication-free, they reported having had multiple hypomanic/manic and/or depressive episodes for many years, consistent with epidemiological studies that note high rates of BP in community samples, with a large proportion not receiving treatment (Merikangas et al, 2011). See Supplementary Material for exclusion criteria.

\section{MRI Acquisition and Preprocessing}

For details see Supplementary Material.

\section{Identification of Disturbed Network Connections}

Time series were extracted from a 195-ROI resting-statebased atlas. Fourteen cerebellum ROIs were removed, because of inconsistent spatial coverage, leaving 181 ROIs. Connectivity matrices were entered as dependent variables into the Network Based Statistic (NBS) toolbox (Zalesky et al, 2010). In NBS, the regression model is first tested for each link, following which a $t$-threshold $(t>3.35)$ is applied across the network to remove unassociated links. Next, clusters of suprathreshold links (links sharing a node with $\geqslant 1$ other cluster links) are identified and the corrected significance of each cluster computed. Specifically, a corrected $p$-value is calculated by comparing observed cluster size (no. of links) against a null distribution of maximal suprathreshold sizes created via permutation (5000 randomizations), resulting in an overall corrected $\alpha<0.05$. For all analyses, linear and quadratic trends in mean DVARS were covaried, along with bipolar subtype.

To identify disturbances related to BP overall, the first analysis compared all BP participants with healthy controls (with a covariate modeling the difference between (hypo) manic and depressed). Several strategies exist to fractionate BP network disturbances into those related to (hypo)manic and depressive symptoms. One strategy is to compare BP patients in (hypo)manic and depressed episodes separately to controls. However, this is associated with reduced power/accuracy, because most patients with (hypo)mania also report some depressive symptoms and vice versa (ie, comparisons do not cleanly separate symptoms). Thus, we used continuous YMRS/HAMD predictors. Specifically, 
a second analysis was carried out with YMRS and HAMD entered simultaneously as continuous predictors (similar to Spielberg et al, 2015a), thus isolating unique variance associated with each set of symptoms. We use the term 'continuous' to mean that actual YMRS/HAMD scores were used as predictors, as opposed to categorical predictors. This does not imply that the YMRS/HAMD distributions were continuous (they were not because of selection criteria).

All participants were included to effectively separate variance unique to YMRS and HAMD and ensure an appropriate multivariate distribution. Although one purpose of these analyses was to fractionate networks observed for BP $v s$ controls, it remained possible that disturbed connections associated specifically with (hypo)manic or depressive symptoms would not emerge in groupwise comparisons. Thus, YMRS/HAMD analyses included all connections. To identify whether BP subtype moderated the relationship between connectivity and YMRS/HAMD, a third analysis included the two-way interactions between subtype and YMRS/HAMD.

To gain insight into the facets of (hypo)mania/depression driving observed findings, we examined the relationship between significant networks and YMRS/HAMD symptom factor scores. For YMRS, we used weights reported in Hanwella and de Silva (2011) to create factor scores for elevated mood, irritability, and thought disorder. For HAMD, we used weights reported in Pancheri et al (2002) to create scores for core depressive symptoms, somatic anxiety, psychic anxiety, and anorexia. For each significant finding, a regression was carried out with the relevant factor scores entered simultaneously as predictors to isolate unique variance associated with each.

\section{Identification of Disturbed Graph Properties}

Connectivity matrices were entered into the Graph Theoretic GLM (GTG) toolbox v.44 (Spielberg et al, 2015a,b), which computes graph properties for each participant (via Brain Connectivity Toolbox; Rubinov and Sporns, 2010). Each matrix was first thresholded to include only positive weights and normalized via division by median weight (excluding zeros) per matrix. Unlike some studies that use higher (and often multiple) density thresholds and binarize networks, we used only one threshold (zero) and retained link weights. This was done because other strategies remove important information (Rubinov and Sporns, 2011) and were used historically because methods were not available for weighted networks. The zero threshold was used because positive and negative weights are typically analyzed separately (Rubinov and Sporns, 2011), and weight normalization was performed, in part, to account for potential bias introduced by thresholding.

Node-specific and mean Clustering Coefficient (indexing Functional Segregation), Global Efficiency (stable variant of Characteristic Path Length; Functional Integration), and Assortativity (Resilience) were examined. Clustering Coefficient reflects the amount of clustering around a node, and mean Clustering Coefficient is the mean across all nodes (indexing global Segregation). Global Efficiency/Assortativity are global network properties. Global Efficiency is the extent to which nodes are connected via the fewest possible paths, reflecting the efficiency of overall network communication.
Assortativity is the extent to which highly connected nodes are linked to other highly connected nodes, reflecting the level of redundancy in communication hubs. Properties were entered as dependent variables in robust regressions in GTG (5000 permutations, same predictors as above).

To limit the number of comparisons, Clustering Coefficient was examined for only the ROI in the equivalent NBS analysis with the highest number of differential connections. An exception was for YMRS, where we also examined right amygdala, given that this region was central to the BP vs HC network. Relationships were tested only for predictors significant in the equivalent NBS analysis.

\section{RESULTS}

\section{Participants}

Seventy-six patients were enrolled, and data from 15 were excluded because of: not meeting scan day HAMD/YMRS criteria $(n=3)$, unreliable/unclear information $(n=3)$, motion $(n=5)$, sleeping during fMRI $(n=1)$, not completing scan $(n=1)$, serious susceptibility artifact $(n=2)$. Close gender/age match was achieved across 30 bipolar (hypo) manic, 30 bipolar depressed, and 30 healthy controls. The remaining (depressed) patient was excluded to maintain gender/age balance. No significant differences were found between BP groups on mean duration off medication (see Supplementary Material for other comparisons).

\section{BP vs Controls}

Disturbances in network connections. BP (vs controls) exhibited hyperconnectivity in a 48-node, 61-link network (corrected $p=0.022$; Figure 1 ), and the largest percentage of differential links (42\%) were with right amygdala.

Disturbances in Graph Properties. BP exhibited worse Global Efficiency and larger Clustering Coefficient for right amygdala (see Table 1).

\section{Fractionation}

Disturbances in network connections. YMRS was associated with hyperconnectivity in a 57-node, 87-link network (corrected $p=0.007$; Figure 2), and the largest percentage of differential links (20\%) were with left posterior superior frontal gyrus (pSFG; Figure 2f). A midbrain (MB) region (encompassing substantia nigra $(\mathrm{SN})$, red nucleus, and ventral tegmental area (VTA); Figure 2d) and right amygdala (Figure 2e) also accounted for relatively high percentages of differential links (18\% and 13\%, respectively). This network overlapped that observed across all BP patients by $50 \%$. Overlap consisted largely of connections with amygdala and with lateral occipital cortex. To gain insight into the particular facets of (hypo)mania driving findings, we examined relationships between network connectivity and YMRS symptom factors. The elevated mood factor predicted unique variance in mean connectivity of the entire YMRS network $(p=0.010)$, along with mean amygdala $(p=0.006)$ and $\mathrm{MB}(p=0.038)$ links in particular. Irritability predicted unique variance in mean connectivity $(p=0.011)$, along with pSFG $(p=0.006)$ links in particular. 
a

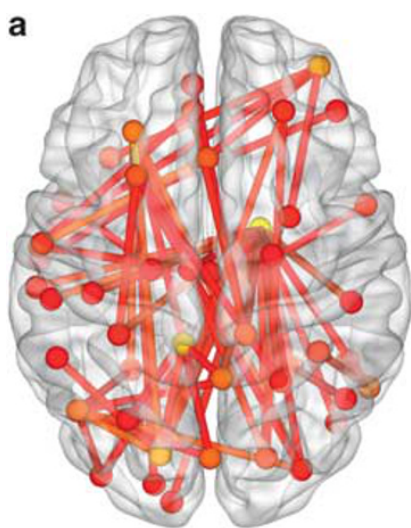

b

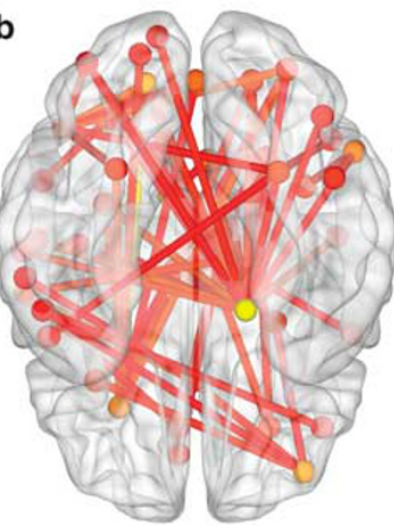

C

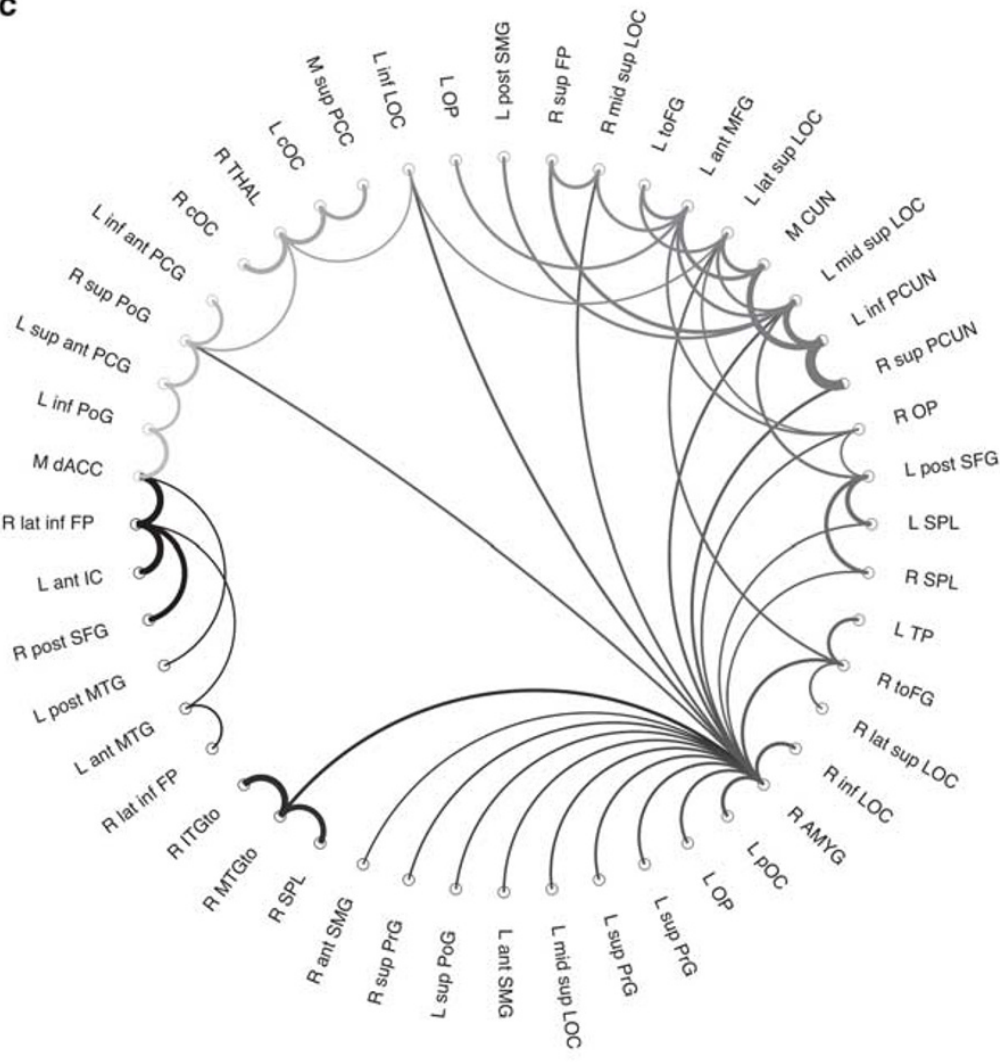

Figure I Network with stronger interconnectivity in bipolar disorder. Network showing stronger interconnectivity across all bipolar patients, relative to healthy controls. ( $a$ and b) Axial views from superior (a) and inferior (b) to brain. Sphere color reflects mean node strength across all participants, and link color reflects the effect size for the relevant test, with the color scales ranging from red (weakest) to yellow (strongest). (c) Connectivity diagram (created via simulated annealing to minimize overlap) where line thickness reflects connectivity strength (mean across all participants). In (c), link color has no particular significance and varies only to assist in visually differentiating between links originating from different nodes. AMYG, amygdala; ant, anterior; cOC, central opercular cortex; $C U N=$ cuneus; $\mathrm{dACC}=$ dorsal anterior cingulate cortex; FP, frontal pole; IC, insular cortex; inf, inferior; ITGto, inferior temporal gyrus, temporooccipital part; L, left; lat, lateral; M, midline; mid, middle (ie, medial to lat on x axis); MFG, middle frontal gyrus; MTG, middle temporal gyrus; MTGto, middle temporal gyrus, temporooccipital part; OP, occipital pole; PCG, paracingulate gyrus; PCUN, precuneus; pOC, parietal opercular cortex; PoG, postcentral gyrus; PrG, precentral gyrus; R, right; SFG, superior frontal gyrus; SMG, supramarginal gyrus; SPL, superior parietal lobule; sup, superior; THAL, thalamus; toFG, temporal-occipital fusiform gyrus; TP, temporal pole.

Table I Graph Property Findings

Node-specific property

Global network properties

Clustering coefficient

\begin{tabular}{|c|c|c|c|c|c|}
\hline & & \\
\hline & Global efficiency & Assortativity & $R$ amygdala & L pSFG & R pmOFC \\
\hline $\mathrm{BP}$ vs HC & $-0.003(0.031)$ & $-0.004(0.305)$ & $0.008(0.027)$ & $\mathrm{N} / \mathrm{A}$ & N/A \\
\hline YMRS & $-0.001(0.030)$ & $0.000(0.796)$ & $0.001(0.036)$ & $0.000(0.275)$ & $N / A$ \\
\hline
\end{tabular}

Abbreviations: BP, bipolar disorder patients; HAMD, Hamilton Depression Rating Scale; HC, healthy controls; L, left; pmOFC, posterior middle orbitofrontal gyrus; pSFG, posterior superior frontal gyrus; R, right; YMRS, Young Mania Rating Scale.

Note: Cell entries are unstandardized $\beta$-values with associated $p$-value within parentheses.

HAMD was associated with hyperconnectivity in a 26-node, 26-link network (corrected $p=0.038$; Figure 3), and the largest percentage of differential links (50\%) were with right posterior-middle orbitofrontal cortex (pmOFC, located in posterior orbital gyrus). This network overlapped the cross-BP network by $8 \%$. Overlap largely consisted of central operculum connections. The somatic anxiety HAMD factor uniquely predicted mean connectivity in the entire HAMD network $(p<0.001)$, along with pmOFC $(p=0.026)$ links in particular.

YMRS and HAMD networks did not overlap. No interactions with BP subtype were observed. 

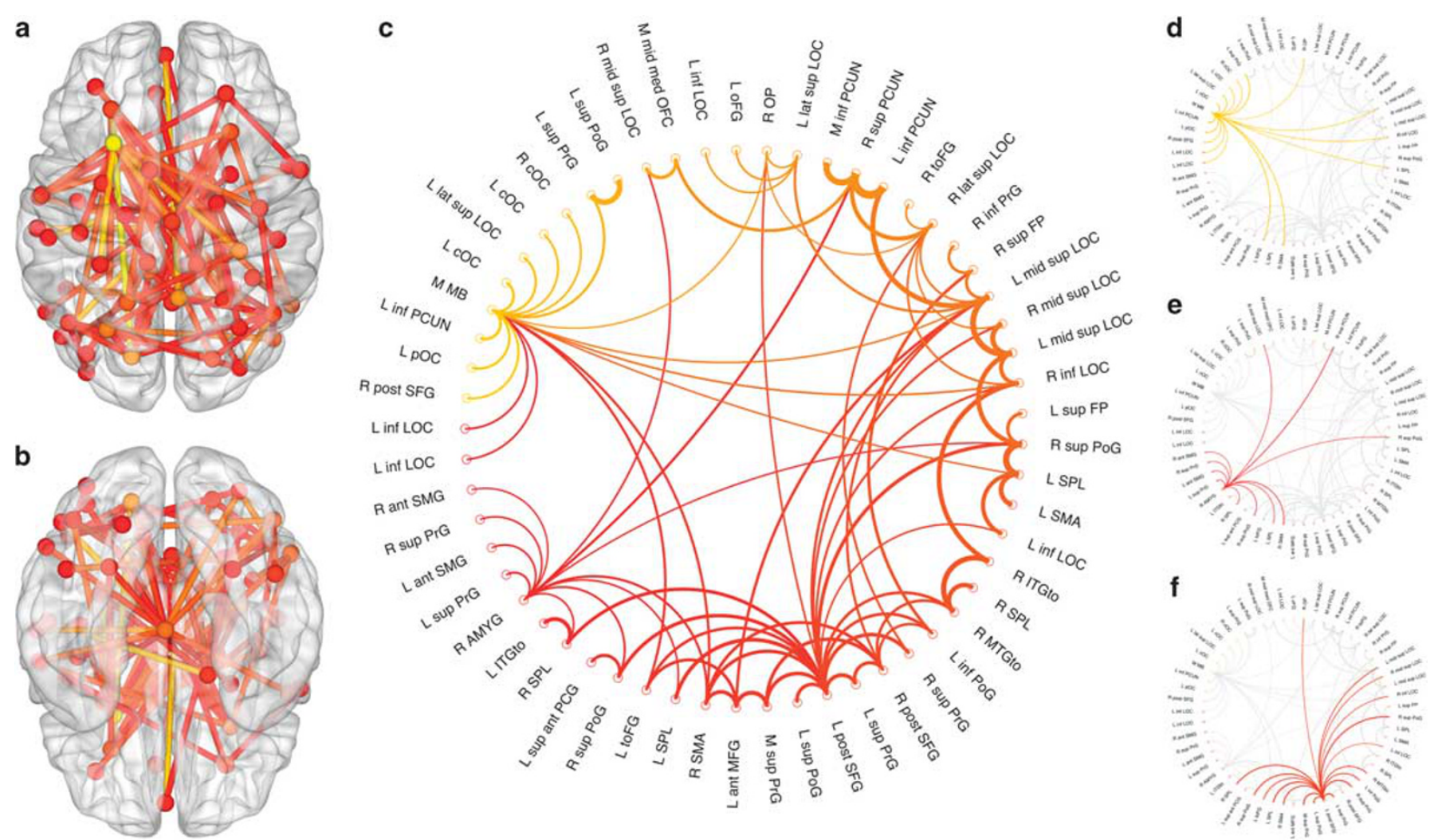

Figure 2 Network with stronger interconnectivity correlating with (hypo)manic symptoms. Network showing stronger interconnectivity with higher values on the Young Mania Rating Scale (YMRS). ( $a$ and b) Axial views from superior (a) and inferior (b) to brain. Sphere color reflects mean node strength across all participants, and link color reflects the effect size for the relevant test, with the color scales ranging from red (weakest) to yellow (strongest). (c) Connectivity diagram (created via simulated annealing to minimize overlap) where line thickness reflects connectivity strength (mean across all participants). In (c-f), link color has no particular significance and varies only to assist in visually differentiating between links originating from different nodes. (d-f) Connectivity diagrams highlighting connections with midbrain, amygdala, and superior frontal gyrus, respectively. AMYG, amygdala; ant, anterior; cOC, central opercular cortex; FP, frontal pole; inf, inferior; ITGto, inferior temporal gyrus, temporooccipital part; L, left; lat, lateral; LOC, lateral occipital cortex; M, midline; med, medial; mid, middle (ie, medial to lat on $\times$ axis); MB, midbrain; MFG, middle frontal gyrus; MTGto, middle temporal gyrus, temporooccipital part; OFC, orbitofrontal cortex; oFG, occipital fusiform gyrus; OP, occipital pole; PCG, paracingulate gyrus; PCUN, precuneus; PoG, postcentral gyrus; PrG, precentral gyrus; R, right; SFG, superior frontal gyrus; SMA, supplementary motor area; SMG, supramarginal gyrus; SPL, superior parietal lobule; sup, superior; toFG, temporal-occipital fusiform gyrus; toFG = temporal-occipital fusiform gyrus.

Disturbances in graph properties. YMRS predicted worse Global Efficiency and larger Clustering Coefficient for right amygdala (see Table 1). The irritability factor uniquely predicted worse Global Efficiency (Table 1). HAMD predicted weaker global Assortativity and larger Clustering Coefficient for right pmOFC (Table 1). The depressed mood factor uniquely predicted weaker Assortativity (Table 1). No findings emerged for mean Clustering Coefficient.

See Supplementary Material for additional analyses.

\section{DISCUSSION}

The aim of the present work was to isolate BP-related disturbances in functional brain networks and fractionate these disturbances into components associated with (hypo) manic and depressive symptoms. Using graph-theory techniques in a sample of medication-free BP patients, we identified a network of connectivity differences present across BP (Figure 1) and fractionated this into segments related to (hypo)manic or depressive symptoms. We also identified symptom-related disturbances in the functional organization of both global and local networks (see Table 1 for a summary of findings).

\section{Disturbances Present Across (Hypo)mania and Depression}

Across BP participants, greater connectivity was observed in a network in which right amygdala had the largest number of differential links (Figure 1). Examination of graph-theory properties further revealed that BP was associated with greater network clustering (Clustering Coefficient, indexing Functional Segregation) around right amygdala, suggesting that amygdala has a greater influence over widespread network processing in BP. These findings are consistent with meta-analyses of activation studies, where amygdala was consistently associated with BP (Chen et al, 2011; Houenou et al, 2011; Delvecchio et al, 2012). In fact, amygdala was one of only two regions consistently observed across extant metaanalyses of BP activation. As evident in Figure 1, the regions showing disturbed connectivity with amygdala are largely in primary, secondary, or higher-order (ie, association) sensory cortices. Given amygdala's central role in determining 

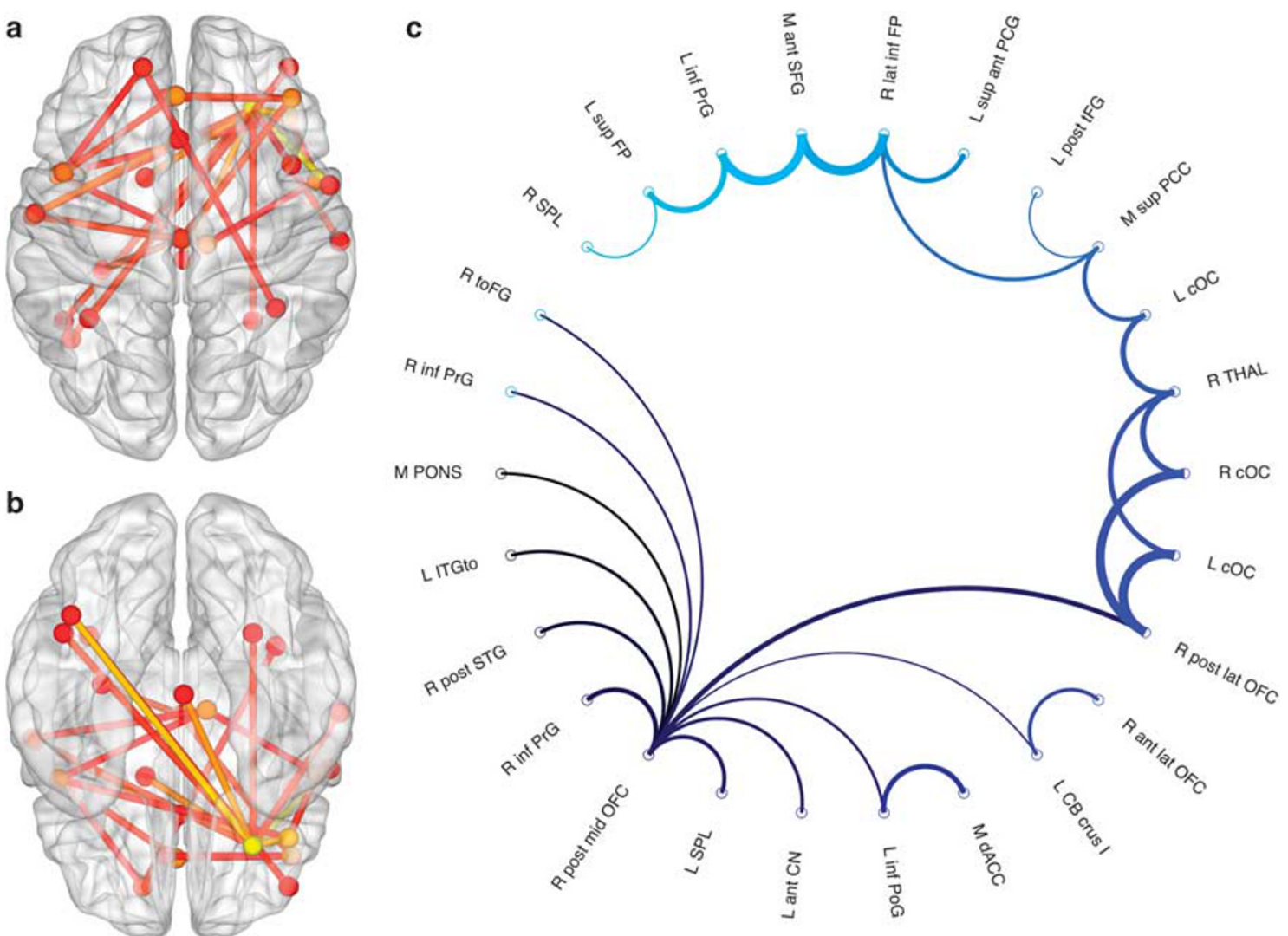

Figure 3 Network with stronger interconnectivity correlating with depressive symptoms. Network showing stronger interconnectivity with higher values on the Hamilton Depression Rating Scale (HAMD). ( $a$ and b) Axial views from superior (a) and inferior (b) to brain. Sphere color reflects mean node strength across all participants, and link color reflects the effect size for the relevant test, with the color scales ranging from red (weakest) to yellow (strongest). (c) Connectivity diagram (created via simulated annealing to minimize overlap) where line thickness reflects connectivity strength (mean across all participants). In (c), link color has no particular significance and varies only to assist in visually differentiating between links originating from different nodes. Abbreviations:; ant = anterior; CB, cerebellum; CN, caudate nucleus; COC, central opercular cortex; dACC, dorsal anterior cingulate cortex; FP, frontal pole; inf, inferior; ITGto, inferior temporal gyrus, temporooccipital part; L, left; lat, lateral; M, midline; mid, middle (ie, medial to lat on $\times$ axis); OFC, orbitofrontal cortex; PCC, posterior cingulate cortex; PCG, paracingulate gyrus; PCUN, precuneus; PoG, postcentral gyrus; PONS, pons; post, posterior; PrG, precentral gyrus; R, right; SFG, superior frontal gyrus; SPL, superior parietal lobule; STG, superior temporal gyrus; sup, superior; tFG, temporal fusiform gyrus; THAL, thalamus; toFG, temporal-occipital fusiform gyrus.

affective salience (Phelps, 2006), hyperconnectivity between amygdala and sensory regions and greater amygdala network clustering may reflect a form of hypersalience, wherein affective significance is inappropriately overlaid on perception.

Present findings of disturbed connectivity with sensory regions may be surprising given that past $\mathrm{BP}$ research and theory often focused on regions more typically linked to affect (Phillips and Swartz, 2014). However, ours is the first study to examine 'whole-brain' connectivity in BP (ie, examination of connectivity between all nodes), rather than limiting to a small subset (eg, 1-3) of regions. Thus, findings similar to ours could have emerged previously had different analysis been used. Also, we identified disturbed connections, which can be with regions that themselves are not 'disturbed', potentially explaining why sensory regions have not emerged in meta-analyses of activation studies.

Consistent with the hypotheses, graph-theory analyses revealed that BP was associated with less efficient global network communication (Global Efficiency, indexing Functional Integration). High integration is crucial for maintaining a 'small-world' network organization (Watts and
Strogatz, 1998), which is found in diverse networks including the brain (Muldoon et al, 2016). A 'small-world' organization is thought to be optimal, because such networks tend to show greater computational power and adaptive reconfiguration (Watts and Strogatz, 1998). Our finding that BP was associated with worse integration suggests that the brain's 'small-world' balance is disturbed in BP.

These analyses captured variance common to BP, but did not isolate disturbances specific to each state. Thus, we examined the relationship between networks and (hypo) manic and depressive symptoms simultaneously, thus isolating the unique variance associated with each state.

\section{Disturbances Related to (Hypo)manic Symptoms}

We identified a network in which (hypo)manic symptoms (YMRS) were related to hyperconnectivity (Figure 2). This network considerably overlapped (by 50\%) that observed across all BP patients and right amygdala again had a large percentage of differential links (Figure 2e). Similar to the findings for all BP patients, YMRS was associated with less efficient global communication and greater amygdala 
network clustering. Thus, although amygdala disturbance and worse overall communication were observed across all $\mathrm{BP}$ patients, these disturbances appear to have a more crucial role in (hypo)mania. As mentioned above, increased connectivity between amygdala and sensory regions may reflect a form of hypersalience. If true, individuals with (hypo)manic symptoms may perceive affective connections where none exist, which may contribute to the elevated mood characteristic of (hypo)mania. This hypothesis is supported by our finding that the elevated mood YMRS symptom factor uniquely predicted amygdala hyperconnectivity.

Along with amygdala, a large percentage of differential links in this network were with a MB region (Figure 2d) encompassing SN, red nucleus, and VTA. Because this node includes multiple structures, we cannot determine which region(s) are driving findings. However, SN and VTA are the brain's primary sources of dopaminergic innervation, and are thus crucial components of reward circuitry (Haber and Knutson, 2010). Thus, it is unsurprising that the elevated mood YMRS factor uniquely predicted MB hyperconnectivity. Thus, (hypo)mania-related increases in the pursuit of pleasurable activities may stem, in part, from hyperactivation of mesocortical/mesolimbic dopamine pathways.

The largest percentage of differential links in the YMRS network was with left pSFG. These connections were primarily with association cortex (eg, superior parietal lobule), and research suggests that such pathways have a crucial role in goal-directed behavior (Banich, 2009). In particular, posterior prefrontal cortex is thought to bias parietal association cortex towards preferential processing of goal-relevant stimuli. Thus, hyperconnectivity between pSFG and association cortex may reflect the excessive goal-striving crucial to (hypo)mania. Combined with research indicating that hindered goal pursuit can lead to irritable mood in both healthy and BP populations (Harmon-Jones, 2003; Urošević et al, 2008), our finding that the YMRS irritability factor uniquely predicted pSFG hyperconnectivity may reflect both increased goal-striving and the frustration of such pursuits.

\section{Disturbances Related to Depressive Symptoms}

We identified a network in which depressive symptoms (HAMD) were associated with hyperconnectivity (Figure 3), and the largest percentage of differential links were with right pmOFC. This network had fairly small (8\%) overlap with the cross-BP network. The observed pattern of HAMD-related hyperconnectivity differs from a recent meta-analysis of resting-state connectivity in MDD (Kaiser et al, 2015). Thus, it is possible that the neural circuitry supporting BP-related depression is fundamentally different from that in MDD. This is consistent with a recent meta-analysis, which found several differences when comparing BP and MDD activation during facial affect paradigms (Delvecchio et al, 2012). The BP patients in this meta-analysis were not necessarily depressed, and thus these findings may reflect differences between mania and depression. However, several activation studies have found differences when directly comparing MDD with depressed BP (Fournier et al, 2013; Lawrence et al, 2004; Rive et al, 2015), and similar studies are needed to directly compare MDD and depressed BP networks.
HAMD was also related to worse global network resilience (Assortativity), suggesting that network processing in depressed BP is more vulnerable to disruption (eg, by distraction). Furthermore, HAMD was associated with greater clustering in the local network surrounding pmOFC (Clustering Coefficient), suggesting that pmOFC has a greater influence over widespread network processing in $\mathrm{BP}$ depression. Given the role of OFC in maintaining the motivational value of stimuli (Kennerley et al, 2011), elevated clustering may relate to the biases in affective value observed in depression (Anderson et al, 2014). Specifically, OFC clustering may reflect more intense/frequent value-related processing, potentially occurring because depressed individuals are less able to discriminate value and attempt to compensate by overengaging OFC. In turn, disturbances in stimulus valuation may be reflected in the difficulty making decisions found in depression.

\section{Fractionation of Network Observed Across BP Patients}

Interestingly, only (hypo)manic symptoms were related to amygdala disturbance, which formed the core of the network observed across all BP patients. Thus, the YMRS network appears to be more analogous to the cross-BP network, consistent with the fact that (hypo)mania is the uniquely defining characteristic of BP. Given that no paths were shared between YMRS and HAMD networks, they may reflect at least partially separable pathophysiological processes. Past work indicates that family transmissions of manic and depressed episodes in BP may be independent, which the authors suggest is reflective of partially distinct biological pathways in mania and depression (Merikangas et al, 2014). Interestingly, both YMRS and HAMD were associated with hyperconnectivity, indicating that transitioning between (hypo)mania and depression is not simply a matter of tuning circuitry up or down.

Although not examined here, BP is also associated with mixed episodes, characterized by both manic and depressive symptoms. Thus, although YMRS and HAMD networks did not overlap in the present study, the brain states associated with these symptoms cannot be exclusive. Instead, there are likely neural processes promoting the onset of both (hypo) manic and depressive symptoms, and present findings should not be interpreted as indicative that the brain states associated with these symptoms operate in an all-or-none manner.

\section{Strengths and Limitations}

The present study benefited from a number of strengths, including a medication-free sample, examination of (hypo) manic and depressed BP patients simultaneously, and use of graph-theory methods. Several limitations must also be considered. Although participants were medication-free, they were not medication-naïve. As such, present findings may be driven, in part, by long-term medication use. Furthermore, this work was cross-sectional and could not differentiate between factors predisposing toward the development of BP from factors arising as a consequence. An ideal study would longitudinally examine the same individuals through both depressive and (hypo)manic episodes. However, such work is extremely difficult and it is unethical to keep patients 
medication-free. Therefore, present findings should be regarded as complementary to studies with medicated participants. In addition, although we can speculate as to the real-world impact of the observed network disturbances, we did not collect behavioral data to confirm these hypotheses. Thus, these hypotheses should be considered preliminary and their veracity examined in future research. As this study was conducted in medication-free outpatients, only two patients met the criteria for a full manic episode (the rest hypomanic) and the majority of the depressed patients were only moderately depressed. Thus, although we examined symptoms continuously, the extent to which present findings are representative of more severe BP (ie, mania, severe depression) remains unknown. Finally, present findings should be considered preliminary until replicated in both resting-state fMRI and other methodologies. For example, task fMRI can probe the dependence of the network disturbances on the affective context.

\section{Summary and Conclusions}

We found network differences present across all BP participants and fractionated these disturbances into components related to (hypo)manic and depressive symptoms. We also found symptom-related differences in the functional organization of the network: (hypo)manic symptoms were related to less efficient communication across the global network, whereas depressive symptoms were associated with a less resilient organization of the global network. Further, both (hypo)manic and depressed symptoms predicted greater local clustering around key nodes in their respective networks.

Present findings provide deeper insight into the pathophysiological processes associated with (hypo)mania and depression. This insight may have key implications for the development and refinement of therapeutic interventions. For example, patients with disturbance primarily in one symptom network may respond differentially to treatment. Thus, quantifying the magnitude of disturbance in each network could prove useful in treatment selection. In summary, we demonstrated that BP-related pathology has important and heterogeneous effects on brain networks and related graph properties. Results move us closer to understanding the precise networks disturbed in BP and highlight the importance of examining the shared and unique network pathology related to (hypo)manic and depressive symptoms.

\section{FUNDING AND DISCLOSURE}

The authors declare no conflict of interest.

\section{ACKNOWLEDGMENTS}

This project was funded by a NIMH grant to AA (R01MH075025).

\section{REFERENCES}

Anand A, Li Y, Wang Y, Gardner K, Lowe MJ (2007). Reciprocal effects of antidepressant treatment on activity and connectivity of the mood regulating circuit: an fMRI study. J Neuropsychiatry Clin Neurosci 19: 274-282.

Anderson BA, Leal SL, Hall MG, Yassa MA, Yantis S (2014). The attribution of value-based attentional priority in individuals with depressive symptoms. Cogn Affect Behav Neurosci 14: 1221-1227.

Anticevic A, Brumbaugh MS, Winkler AM, Lombardo LE, Barrett J, Corlett PR et al (2013). Global prefrontal and fronto-amygdala dysconnectivity in bipolar I disorder with psychosis history. Biol Psychiatry 73: 565-573.

Banich MT (2009). Executive function the search for an integrated account. Curr Direct Psychol Sci 18: 89-94.

Blumberg HP (2012). Euthymia, depression, and mania: what do we know about the switch? Biol Psychiatry 71: 570-571.

Brady R Jr, Öngür D, Keshavan M (2014). Neurobiology of moodstate shifts in bipolar disorder: a selective review and a hypothesis. Harvard Rev Psychiatry 22: 23-30.

Chai XJ, Whitfield-Gabrieli S, Shinn AK, Gabrieli JD, Castañón AN, McCarthy JM et al (2011). Abnormal medial prefrontal cortex resting-state connectivity in bipolar disorder and schizophrenia. Neuropsychopharmacology 36: 2009-2017.

Chen CH, Suckling J, Lennox BR, Ooi C, Bullmore ET (2011). A quantitative meta-analysis of fMRI studies in bipolar disorder. Bipolar Disord 13: 1-5.

Delvecchio G, Fossati P, Boyer P, Brambilla P, Falkai P, Gruber O et al (2012). Common and distinct neural correlates of emotional processing in bipolar disorder and major depressive disorder: a voxel-based meta-analysis of functional magnetic resonance imaging studies. Eur Neuropsychopharmacol 22: 100-113.

Fournier JC, Keener MT, Almeida J, Kronhaus DM, Phillips ML (2013). Amygdala and whole-brain activity to emotional faces distinguishes major depressive disorder and bipolar disorder. Bipolar Disord 15: 741-752.

Haber SN, Knutson B (2010). The reward circuit: linking primate anatomy and human imaging. Neuropsychopharmacology 35: $4-26$.

Hamilton M (1960). A rating scale for depression. J Neurol Neurosurg Psychiatry 23: 56.

Hanwella R, de Silva VA (2011). Signs and symptoms of acute mania: a factor analysis. BMC Psychiatry 11: 137.

Harmon-Jones E (2003). Anger and the behavioral approach system. Person Individ Differ 35: 995-1005.

Houenou J, Frommberger J, Carde S, Glasbrenner M, Diener C, Leboyer $\mathrm{M}$ et al (2011). Neuroimaging-based markers of bipolar disorder: evidence from two meta-analyses. J Affect Disord 132: 344-355.

Kaiser RH, Andrews-Hanna JR, Wager TD, Pizzagalli DA (2015). Large-scale network dysfunction in major depressive disorder: a meta-analysis of resting-state functional connectivity. JAMA Psychiatry 72: 687-696.

Kennerley SW, Behrens TE, Wallis JD (2011). Double dissociation of value computations in orbitofrontal and anterior cingulate neurons. Nat Neurosci 14: 1581-1589.

Lawrence NS, Williams AM, Surguladze S, Giampietro V, Brammer MJ, Andrew C et al (2004). Subcortical and ventral prefrontal cortical neural responses to facial expressions distinguish patients with bipolar disorder and major depression. Biol Psychiatry 55: 578-587.

Leow A, Ajilore O, Zhan L, Arienzo D, GadElkarim J, Zhang A et al (2013). Impaired inter-hemispheric integration in bipolar disorder revealed with brain network analyses. Biol Psychiatry 73: 183-193.

Merikangas KR, Cui L, Heaton L, Nakamura E, Roca C, Ding J et al (2014). Independence of familial transmission of mania and depression: results of the NIMH family study of affective spectrum disorders. Mol Psychiatry 19: 214-219.

Merikangas KR, Jin R, He JP, Kessler RC, Lee S, Sampson NA et al (2011). Prevalence and correlates of bipolar spectrum disorder in 
the world mental health survey initiative. Archiv Gen Psychiatry 68: 241-251.

Muldoon SF, Bridgeford EW, Bassett DS (2016). Small-world propensity and weighted brain networks. Scientific Rep 6: 22057.

Öngür D, Lundy M, Greenhouse I, Shinn AK, Menon V, Cohen BM et al (2010). Default mode network abnormalities in bipolar disorder and schizophrenia. Psychiatry Res 183: 59-68.

Pancheri P, Picardi A, Pasquini M, Gaetano P, Biondi M (2002). Psychopathological dimensions of depression: a factor study of the 17-item Hamilton depression rating scale in unipolar depressed outpatients. J Affect Disord 68: 41-47.

Phelps EA (2006). Emotion and cognition: insights from studies of the human amygdala. Annual Review of Psychology 57: 27-53.

Phillips ML, Swartz HA (2014). A critical appraisal of neuroimaging studies of bipolar disorder: toward a new conceptualization of underlying neural circuitry and a road map for future research. Am J Psychiatry 171: 829-843.

Rive MM, Mocking RJ, Koeter MW, van Wingen G, de Wit SJ, van den Heuvel OA et al (2015). State-dependent differences in emotion regulation between unmedicated bipolar disorder and major depressive disorder. JAMA Psychiatry 72: 603-611.

Rubinov M, Sporns O (2010). Complex network measures of brain connectivity: uses and interpretations. Neuroimage 52: 1059-1069.

Rubinov M, Sporns O (2011). Weight-conserving characterization of complex functional brain networks. Neuroimage 56: 2068-2079.

Sheehan DV, Lecrubier Y, Sheehan KH, Amorim P, Janavs J, Weiller E et al (1998). The Mini-International Neuropsychiatric Interview (MINI): the development and validation of a structured diagnostic psychiatric interview for DSM-IV and ICD-10. J Clin Psychiatry 59: 22-33.
Spielberg JM, McGlinchey RE, Milberg WP, Salat DH (2015a). Brain network disturbance related to posttraumatic stress \& traumatic brain injury in veterans. Biol Psychiatry 78: 210-216.

Spielberg JM, Miller GA, Heller W, Banich MT (2015b). Flexible brain network reconfiguration supporting inhibitory control. Proc Natl Acad Sci USA 112: 10020-10025.

Strakowski SM, Adler CM, Almeida J, Altshuler LL, Blumberg HP, Chang KD et al (2012). The functional neuroanatomy of bipolar disorder: a consensus model. Bipolar Disord 14: 313-325.

Torrisi S, Moody TD, Vizueta N, Thomason ME, Monti MM, Townsend JD et al (2013). Differences in resting corticolimbic functional connectivity in bipolar I euthymia. Bipolar Disord 15: $156-166$.

Urošević S, Abramson LY, Harmon-Jones E, Alloy LB (2008). Dysregulation of the behavioral approach system (BAS) in bipolar spectrum disorders: review of theory and evidence. Clin Psychol Rev 28: 1188-1205.

Vargas C, López-Jaramillo C, Vieta E (2013). A systematic literature review of resting state network-functional MRI in bipolar disorder. J Affect Disord 150: 727-735.

Watts DJ, Strogatz SH (1998). Collective dynamics of 'small-world' networks. Nature 393: 440-442.

Wessa M, Kanske P, Linke J (2014). Bipolar disorder: a neural network perspective on a disorder of emotion and motivation. Restor Neurol Neurosci 32: 51-62.

Young RC, Biggs JT, Ziegler VE, Meyer DA (1978). A rating scale for mania: reliability, validity and sensitivity. Br J Psychiatry 133: 429-435.

Zalesky A, Fornito A, Bullmore ET (2010). Network-based statistic: identifying differences in brain networks. Neuroimage 53: 1197-1207.

Supplementary Information accompanies the paper on the Neuropsychopharmacology website (http://www.nature.com/npp) 